\title{
Note on the Hahn-Banach Theorem in a Partially Ordered Vector Space
}

\author{
Toshiharu Kawasaki, ${ }^{1}$ Masashi Toyoda, ${ }^{2}$ and Toshikazu Watanabe ${ }^{3}$ \\ ${ }^{1}$ College of Engineering, Nihon University, Naka-gawara, Tokusada, Tamura-machi, Koriyama-shi, Fukushima 963-8642, Japan \\ ${ }^{2}$ Faculty of Engineering, Tamagawa University, 6-1-1 Tamagawa-gakuen, Machida-shi, Tokyo 194-8610, Japan \\ ${ }^{3}$ Graduate School of Science and Technology, Niigata University, 8050 Ikarashi 2-no-cho, Nishi-ku, Niigata 950-2181, Japan
}

Correspondence should be addressed to Masashi Toyoda; mss-toyoda@eng.tamagawa.ac.jp

Received 17 May 2013; Accepted 25 June 2013

Academic Editor: Wei-Shih Du

Copyright (C) 2013 Toshiharu Kawasaki et al. This is an open access article distributed under the Creative Commons Attribution License, which permits unrestricted use, distribution, and reproduction in any medium, provided the original work is properly cited.

Using a fixed point theorem in a partially ordered set, we give a new proof of the Hahn-Banach theorem in the case where the range space is a partially ordered vector space.

\section{Introduction}

The Hahn-Banach theorem is one of the most fundamental theorems in the functional analysis theory. This theorem is well known in the case where the range space is the real number system as follows.

Let $p$ be a sublinear mapping from a vector space $X$ into the real number system $R, Y$ a subspace of $X$, and $q$ a linear mapping from $Y$ into $R$ such that $q \leq p$ on $Y$. Then there exists a linear mapping $g$ from $X$ into $R$ such that $g=q$ on $Y$ and $g \leq p$ on $X$.

It is known that this theorem is established in the case where the range space is a Dedekind complete Riesz space as follows [1-3].

Let $p$ be a sublinear mapping from a vector space $X$ into a Dedekind complete Riesz space $E, Y$ a subspace of $X$ and $q$ a linear mapping from $Y$ into $E$ such that $q \leq p$ on $Y$. Then there exists a linear mapping $g$ from $X$ into $E$ such that $g=q$ on $Y$ and $g \leq p$ on $X$.

On the other hand, Hirano et al. [4] showed the HahnBanach theorem by using the Markov-Kakutani fixed point theorem [5] in the case where the range space is the real number system.

In this paper, motivated by Hirano et al. [4], we give a proof of the Hahn-Banach theorem using a fixed point theorem. We show the Hahn-Banach theorem in the case where the range space is a Dedekind complete partially ordered vector space (Theorem 10). Moreover, we show the Mazur-Orlicz theorem in a Dedekind complete partially ordered vector space (Theorem 11).

\section{Preliminaries}

Let $(E, \leq)$ be a partially ordered set and $F$ a subset of $E$. The set $F$ is called a chain if any two elements are comparable; that is, $x \leq y$ or $y \leq x$ for any $x, y \in F$. An element $x \in E$ is called a lower bound of $F$ if $x \leq y$ for any $y \in F$. An element $x \in E$ is called the minimum of $F$ if $x$ is a lower bound of $F$ and $x \in F$. If there exists a lower bound of $F$, then $F$ is said to be bounded from below. An element $x \in E$ is called an upper bound of $F$ if $y \leq x$ for any $y \in F$. An element $x \in E$ is called the maximum of $F$ if $x$ is an upper bound and $x \in F$. If there exists an upper bound of $F$, then $F$ is said to be bounded from above. If the set of all lower bounds of $F$ has the maximum, then the maximum is called an infimum of $F$ and denoted by inf $F$. If the set of all upper bounds of $F$ has the minimum, then the minimum is called a supremum of $F$ and denoted by sup $F$. An element $x \in F$ is called a minimal of $F$ if $y \leq x$ and $y \in F$ implies $y=x$. A partially ordered set $E$ is said to be complete if every nonempty chain of $E$ has an infimum; $E$ is said to be chain complete if every nonempty chain of $E$ 
which is bounded from below has an infimum; $E$ is said to be Dedekind complete if every nonempty subset of $E$ which is bounded from below has an infimum. A mapping $f$ from $E$ into $E$ is said to be decreasing if $f(x) \leq x$ for any $x \in E$. For further information of a partially ordered set, see [1, 2, 6-10].

In a complete partially ordered set, the following theorem is obtained; see [11-14].

Theorem 1 (Bourbaki-Kneser). Let $E$ be a complete partially ordered set. Let $f$ be a decreasing mapping from $E$ into $E$. Then $f$ has a fixed point.

A partially ordered set $E$ is called a partially ordered vector space if $E$ is a vector space and $x+z \leq y+z$ and $\alpha x \leq \alpha y$ hold whenever $x, y, z \in E, x \leq y$ and $\alpha$ is a nonnegative real number. If a partially ordered vector space $E$ is a lattice, that is, any two elements in $E$ have a supremum and an infimum, then $E$ is called a Riesz space.

Let $X$ be a vector space and $E$ a partially ordered vector space. A mapping $f$ from $X$ into $E$ is said to be concave if $f(t x+(1-t) y) \geq t f(x)+(1-t) f(y)$ for any $x, y \in X$ and $t \in[0,1]$. A mapping $p$ from $X$ into $E$ is said to be sublinear if the following conditions are satisfied.

(S1) For any $x, y \in X, p(x+y) \leq p(x)+p(y)$.

(S2) For any $x \in X$ and nonnegative real number $\alpha$, $p(\alpha x)=\alpha p(x)$.

Let $E^{X}$ be the set of mappings from $X$ into $E$. Throughout this paper, $E^{X}$ is ordered as follows. For $f, g \in E^{X}$, let $f \leq g$ mean that $f(x) \leq g(x)$ for any $x \in X$. It is easy to check that $E^{X}$ is also a partially ordered vector space.

The following lemmas are useful for the proof of our main results.

Lemma 2. Let $X$ be a vector space, $E$ a chain complete partially ordered vector space, and $Z$ a nonempty chain of $E^{X}$ which is bounded from below. Then there exists $\inf \{h(x) \mid h \in Z\}$ for any $x \in X$. Moreover, if $p \in E^{X}$ is defined by $p(x)=\inf \{h(x) \mid$ $h \in Z\}$ for any $x \in X$, then $p=\inf Z$; that is, $E^{X}$ is chain complete.

Proof. Let $x \in X$ be fixed. Since $Z$ is a nonempty chain, so is $\{h(x) \mid h \in Z\}$. Let $f$ be a lower bound of $Z$. Since $f(x) \leq$ $h(x)$ for any $h \in Z,\{h(x) \mid h \in Z\}$ is bounded from below. Therefore, since $E$ is chain complete, there exists $\inf \{h(x)$ | $h \in Z$.

Define $p \in E^{X}$ by $p(x)=\inf \{h(x) \mid h \in Z\}$ for any $x \in X$. Then it is clear that $p \leq h$ for any $h \in Z$; that is, $p$ is a lower bound of $Z$. Let $q$ be a lower bound of $Z$. Since $q(x) \leq h(x)$ for any $x \in X$ and $h \in Z, q(x)$ is a lower bound of $\{h(x) \mid h \in Z\}$ for any $x \in X$. Therefore, $q(x) \leq \inf \{h(x) \mid h \in Z\}=p(x)$ for any $x \in X$ and thus $p=\inf Z$.

Lemma 3. Let $X$ be a vector space, $E$ a Dedekind complete partially ordered vector space, and $Z$ a nonempty subset in $E^{X}$ which is bounded from below. Then there exists $\inf \{h(x) \mid$ $h \in Z\}$ for any $x \in X$. Moreover, if $p \in E^{X}$ is defined by $p(x)=\inf \{h(x) \mid h \in Z\}$ for any $x \in X$, then $p=\inf Z$; that is, $E^{X}$ is Dedekind complete.

Proof. The proof is similar to that of Lemma 2.

Lemma 4. Let $X, E, E^{X}, Z$, and $p$ be the same as in Lemma 2. Suppose that

(1) for any $h \in Z, x \in X$ and $\alpha>0$, there exists $h^{\prime} \in Z$ such that $h(\alpha x)=\alpha h^{\prime}(x)$;

(2) $p(0)=0$;

(3) for any $h_{1}, h_{2} \in Z$ and $x, y \in X$, there exists $h \in Z$ such that $h(x+y) \leq h_{1}(x)+h_{2}(y)$.

Then $p$ is sublinear.

Proof. Let $x \in X$ and $\alpha>0$ be fixed. It is clear from (1) that $\{h(\alpha x) \mid h \in Z\} \subset\left\{\alpha h^{\prime}(x) \mid h^{\prime} \in Z\right\}$. Since $\alpha x \in X$ and $1 / \alpha>0$, by (1), for any $h^{\prime} \in Z$ there exists $h \in Z$ such that

$$
\alpha h^{\prime}(x)=\alpha h^{\prime}\left(\frac{1}{\alpha} \alpha x\right)=\alpha \frac{1}{\alpha} h(\alpha x)=h(\alpha x)
$$

and hence $\left\{\alpha h^{\prime}(x) \mid h^{\prime} \in Z\right\} \subset\{h(\alpha x) \mid h \in Z\}$. Therefore, we conclude that $\{h(\alpha x) \mid h \in Z\}=\left\{\alpha h^{\prime}(x) \mid h^{\prime} \in Z\right\}$. Thus we obtain that

$$
\begin{aligned}
p(\alpha x) & =\inf \{h(\alpha x) \mid h \in Z\}=\inf \left\{\alpha h^{\prime}(x) \mid h^{\prime} \in Z\right\} \\
& =\alpha \inf \left\{h^{\prime}(x) \mid h^{\prime} \in Z\right\}=\alpha p(x) .
\end{aligned}
$$

Moreover, (2) shows that $p(0 x)=p(0)=0=0 p(x)$. Therefore, (S2) holds.

Let $x, y \in X$ be fixed. By (3), for any $h_{1}, h_{2} \in Z$, there exists $h \in Z$ such that $h(x+y) \leq h_{1}(x)+h_{2}(y)$. Thus we have

$$
p(x+y) \leq h_{1}(x)+h_{2}(y)
$$

for any $h_{1}, h_{2} \in Z$. This shows that $p(x+y)-h_{2}(y)$ is a lower bound of $\{h(x) \mid h \in Z\}$ for any $h_{2} \in Z$ and hence we have

$$
p(x+y)-h_{2}(y) \leq p(x)
$$

for any $h_{2} \in Z$. This shows that $p(x+y)-p(x)$ is a lower bound of $\{h(y) \mid h \in Z\}$ and hence we have $p(x+y)-p(x) \leq p(y)$. Therefore, (S1) holds. This completes the proof.

Lemma 5. Let $X, E, E^{X}, Z$, and $p$ be the same as in Lemma 3. Suppose that

(1) for any $h \in Z, x \in X$ and $\alpha>0$, there exists $h^{\prime} \in Z$ such that $h(\alpha x)=\alpha h^{\prime}(x)$;

(2) $p(0)=0$;

(3) for any $h_{1}, h_{2} \in Z$ and $x, y \in X$, there exists $h \in Z$ such that $h(x+y) \leq h_{1}(x)+h_{2}(y)$.

Then $p$ is sublinear.

Proof. The proof is similar to that of Lemma 4. 


\section{Main Results}

To obtain our main results, we need the following.

Lemma 6. Let $g$ be a sublinear mapping from a vector space $X$ into a chain complete partially ordered vector space $E$ and $y \in X$. Let $\phi$ be a mapping from $X$ into $E$ defined by

$$
\phi(x)=\inf \{g(x+t y)-g(t y) \mid t \geq 0\}
$$

for any $x \in X$. Then $\phi$ is sublinear and $g^{*} \leq \phi \leq g$ on $X$, where $g^{*}$ is a mapping from $X$ into E defined by $g^{*}(x)=-g(-x)$ for $x \in X$.

Proof. For any $x \in X$ and $t \geq 0$, put $\tau_{t}(x)=g(x+t y)-g(t y)$. Then $Z=\left\{\tau_{t} \mid t \geq 0\right\}$ is a nonempty chain and bounded from below in $E^{X}$. Indeed, since $g=\tau_{0} \in Z, Z$ is nonempty. If $s \leq t$, then

$$
\begin{aligned}
\tau_{s}(x)-\tau_{t}(x) & =g(x+s y)-g(s y)-(g(x+t y)-g(t y)) \\
& =g(x+s y)+(g(t y)-g(s y))-g(x+t y) \\
& =g(x+s y)+(t-s) g(y)-g(x+t y) \\
& =g(x+s y)+g((t-s) y)-g(x+t y) \\
& \geq g(x+s y+(t-s) y)-g(x+t y)=0
\end{aligned}
$$

for any $x \in X$. Thus $Z$ is a chain in $E^{X}$. Since

$$
\begin{aligned}
\tau_{t}(x)= & g(x+t y)-g(t y) \geq g(t y) \\
& -g(-x)-g(t y)=-g(-x)=g^{*}(x)
\end{aligned}
$$

for any $x \in X$ and $t \geq 0, g^{*}$ is a lower bound of $Z$. Hence $Z$ is bounded from below in $E^{X}$. Lemma 2 shows that $\phi(x)=$ $\inf Z$ is well defined.

We next check (1), (2), and (3) in Lemma 4. Let $t \geq 0$, $x \in X$, and $\alpha>0$. We have

$$
\begin{aligned}
\tau_{t}(\alpha x) & =g(\alpha x+t y)-g(t y) \\
& =\alpha\left(g\left(x+\frac{t}{\alpha} y\right)-g\left(\frac{t}{\alpha} y\right)\right) \\
& =\alpha \tau_{t / \alpha}(x) .
\end{aligned}
$$

Clearly, $\tau_{t / \alpha} \in Z$ and hence (1) in Lemma 4 holds. Since $\phi(0)=\inf \{0 \mid t \geq 0\}=0,(2)$ in Lemma 4 holds. Let $t_{1}, t_{2} \geq 0$ and $x_{1}, x_{2} \in X$. Since we have

$$
\begin{aligned}
\tau_{t_{1}+t_{2}}\left(x_{1}+x_{2}\right)= & g\left(x_{1}+x_{2}+\left(t_{1}+t_{2}\right) y\right) \\
& -g\left(\left(t_{1}+t_{2}\right) y\right) \leq g\left(x_{1}+t_{1} y\right) \\
& +g\left(x_{2}+t_{2} y\right)-\left(t_{1}+t_{2}\right) g(y) \\
= & g\left(x_{1}+t_{1} y\right)-g\left(t_{1} y\right) \\
& +g\left(x_{2}+t_{2} y\right)-g\left(t_{2} y\right) \\
= & \tau_{t_{1}}\left(x_{1}\right)+\tau_{t_{2}}\left(x_{2}\right),
\end{aligned}
$$

(3) in Lemma 4 holds. Therefore, Lemma 4 implies that $\phi$ is sublinear.

Finally, it is clear that $\phi \leq g$. This inequality and (7) imply that $g^{*} \leq \phi \leq g$ on $X$.

By Theorem 1 and Lemma 6, we obtain the following. For the case that $E$ is a Dedekind complete Riesz space, see [2].

Theorem 7. Let $f$ be a sublinear mapping from a vector space $X$ into a chain complete partially ordered vector space $E$. Then there exists a linear mapping $g$ from $X$ into $E$ such that $g \leq f$ on $X$.

Proof. Let $Y$ be a subset of $E^{X}$ defined by

$$
Y=\left\{h \in E^{X} \mid h \text { is sublinear, } f^{*} \leq h \leq f\right\},
$$

where $f^{*}$ is defined by $f^{*}(x)=-f(-x)$ for any $x \in X$. Then it is clear that $f \in Y$ and hence $Y$ is nonempty. Moreover $Y$ is complete. In fact, let $Z \subset Y$ be a nonempty chain. Since for any $h \in Z, f^{*} \leq h, Z$ is bounded from below. It follows from Lemma 2 that there exists inf $Z \in E^{X}$. By Lemma 4, $\inf Z$ is sublinear. Since $f^{*} \leq h \leq f$ for any $h \in Z$, we have $f^{*} \leq \inf Z \leq f$. Thus inf $Z \in Y$ and hence $Y$ is complete. Furthermore $Y$ has a minimal. In fact, we suppose that $Y$ does not have a minimal element. Then, for any $h \in Y$, there exists $\widehat{h} \in Y$ such that $\widehat{h} \leq h$ and $\widehat{h} \neq h$. We define a mapping $T$ from $Y$ into $Y$ by $T h=\widehat{h}$. Since the mapping $T$ is decreasing, there exists $h_{0} \in Y$ satisfying $T h_{0}=h_{0}$ by Theorem 1 . This is a contradiction.

Let $g$ be a minimal in $Y$. Let $x \in X$. Let $\phi$ be a mapping from $X$ into $E$ defined by

$$
\phi(z)=\inf \{g(z+t x)-g(t x) \mid t \geq 0\}
$$

for any $z \in X$, then $\phi$ is sublinear and $g^{*} \leq \phi \leq g$ on $X$ by Lemma 6. Moreover $\phi \in Y$. In fact, since $g \leq f$ and $f^{*} \leq g^{*}$, we have $f^{*} \leq g^{*} \leq \phi \leq g \leq f$ for any $f \in Z$. This shows that $\phi \in Y$. Since $g$ is minimal, $\phi=g$. Then we have

$$
\begin{aligned}
g(-x) & =\phi(-x) \\
& =\inf \{g(-x+t x)-g(t x) \mid t \geq 0\} \\
& \leq g(-x+x)-g(x) \\
& =g(0)-g(x)=-g(x) .
\end{aligned}
$$

Since $g$ is sublinear and $0=g(0) \leq g(x+z)+g(-x-z)$, we have

$$
\begin{aligned}
-g(x+z) & \leq g(-x-z) \\
& \leq g(-x)+g(-z) \\
& \leq-g(x)-g(z) .
\end{aligned}
$$

Thus $g(x)+g(z) \leq g(x+z)$. Since $g$ is sublinear, we also have $g(x+z) \leq g(x)+g(z)$ for any $x, z \in X$. Then we obtain that for any $x, z \in X, g(x+z)=g(x)+g(z)$. Let $x \in X$ and $\alpha>0$. Since

$$
0=g(\alpha x-\alpha x)=\alpha g(x)+g(-\alpha x),
$$


we have $g(-\alpha x)=-\alpha g(x)$. Then for any real number $\alpha$, we have $g(\alpha x)=\alpha g(x)$. Thus $g$ is linear. Therefore, $g$ is a linear mapping from $X$ into $E$ such that $g \leq f$ on $X$.

Since Dedekind completeness implies chain completeness, we obtain the following.

Corollary 8. Let $f$ be a sublinear mapping from a vector space $X$ into a Dedekind complete partially ordered vector space $E$. Then there exists a linear mapping $g$ from $X$ into $E$ such that $g \leq f$ on $X$.

To give the Hahn-Banach Theorem in the case where the range space is a Dedekind complete partially ordered vector space, we need the following.

Lemma 9. Let $p$ be a sublinear mapping from a vector space $X$ into a Dedekind complete partially ordered vector space $E, K$ a nonempty convex subset of $X$, and $q$ a concave mapping from $K$ into $E$ such that $q \leq p$ on $K$. For any $x \in X$, let

$$
\phi(x)=\inf \{p(x+t y)-t q(y) t \geq 0, y \in K\} .
$$

Then $\phi$ is a sublinear mapping such that $\phi \leq p$ on $X$. Moreover, if $g$ is a linear mapping from $X$ into $E$, then $g \leq \phi$ on $X$ is equivalent to $g \leq p$ on $X$ and $q \leq g$ on $K$.

Proof. First, we show that $\phi$ is well defined and $\phi(x) \geq$ $-p(-x)$ for any $x \in X$. Let $Z=\left\{\tau_{t, y} \mid t \geq 0\right.$ and $\left.y \in K\right\}$, where

$$
\tau_{t, y}(x)=p(x+t y)-t q(y)
$$

for any $x \in X$ and $t \geq 0$. For any $\tau_{t, y} \in Z$ and $x \in X$,

$$
\begin{aligned}
\tau_{t, y}(x) & =p(x+t y)-t q(y) \\
& \geq p(t y)-p(-x)-t q(y) \geq-p(-x),
\end{aligned}
$$

and thus $\phi(x) \geq-p(-x)$ and $Z$ is bounded from below in $E^{X}$. Since $E$ is Dedekind complete, $\phi$ is well defined by Lemma 3 .

We next check (1), (2), and (3) in Lemma 5.

(1) Let $\tau_{t, y} \in Z$. For any $x \in X$ and $\alpha>0$, we have

$$
\begin{aligned}
\tau_{t, y}(\alpha x) & =p(\alpha x+t y)-t q(y) \\
& =\alpha\left(p\left(x+\frac{t}{\alpha} y\right)-\frac{t}{\alpha} q(y)\right) \\
& =\alpha \tau_{t / \alpha, y}(x) .
\end{aligned}
$$

(2) By the definition of $\phi, \phi(x) \leq p(x)$ for any $x \in X$. Therefore $\phi(0) \leq p(0)=0$. Since $p \geq q$ on $K$, we have

$$
\begin{aligned}
\phi(0) & =\inf \{p(t y)-t q(y) t \geq 0, y \in K\} \\
& =\inf \{t p(y)-t q(y) t \geq 0, y \in K \geq 0\} .
\end{aligned}
$$

Hence we have $\phi(0)=0$.
(3) Let $\tau_{t_{1}, y_{1}}, \tau_{t_{2}, y_{2}} \in Z$ satisfying $t_{1}+t_{2} \neq 0$. Let $x_{1}, x_{2} \in X$. Since $K$ is convex and $q$ is concave, we have

$$
\begin{aligned}
\tau_{t_{1}, y_{1}} & \left(x_{1}\right)+\tau_{t_{2}, y_{2}}\left(x_{2}\right) \\
& =p\left(x_{1}+t_{1} y_{1}\right)-t_{1} q\left(y_{1}\right)+p\left(x_{2}+t_{2} y_{2}\right)-t_{2} q\left(y_{2}\right) \\
& \geq p\left(x_{1}+x_{2}+\left(t_{1}+t_{2}\right) w\right)-\left(t_{1}+t_{2}\right) q(w) \\
& =\tau_{t_{1}+t_{2}, w}\left(x_{1}+x_{2}\right)
\end{aligned}
$$

where $w=\left(1 /\left(t_{1}+t_{2}\right)\right)\left(t_{1} y_{1}+t_{2} y_{2}\right) \in K$. Since $p$ is sublinear, we have

$$
\begin{aligned}
\tau_{0, w}\left(x_{1}+x_{2}\right) & =p\left(x_{1}+x_{2}\right) \leq p\left(x_{1}\right)+p\left(x_{2}\right) \\
& =\tau_{0, y_{1}}\left(x_{1}\right)+\tau_{0, y_{2}}\left(x_{2}\right) .
\end{aligned}
$$

Therefore, for any $x_{1}, x_{2} \in X$ and $t_{1}, t_{2} \geq 0$, we have $\tau_{t_{1}, y_{1}}\left(x_{1}\right)+\tau_{t_{2}, y_{2}}\left(x_{2}\right) \geq \tau_{t_{1}+t_{2}, w}\left(x_{1}+x_{2}\right)$.

Thus by Lemma $5, \phi$ is sublinear. Moreover, by the definition of $\phi$, we have $\phi \leq p$ on $X$.

Let $g$ be a linear mapping from $X$ into $E$. Suppose that $g \leq \phi$ on $X$. Since $\phi \leq p$ on $X$, we have $g \leq p$ on $X$. Moreover, since for any $y \in K$,

$$
\begin{aligned}
-g(y) & =g(-y) \leq \phi(-y) \\
& \leq p(-y+y)-q(y)=-q(y),
\end{aligned}
$$

we have $g \geq q$ on $K$. To prove the converse, suppose that $g \leq p$ on $X$ and $q \leq g$ on $K$. For any $x \in X, y \in K$ and $t \geq 0$, we have

$$
g(x)=g(x+t y)-\operatorname{tg}(y) \leq p(x+t y)-t q(y) .
$$

This implies that $g \leq \phi$ on $X$.

By Corollary 8 and Lemma 9, we have the Hahn-Banach theorem in the case where the range space is a Dedekind complete partially ordered vector space. For the case that $E$ is a Dedekind complete Riesz space, see [2].

Theorem 10. Let $p$ be a sublinear mapping from a vector space $X$ into a Dedekind complete partially ordered vector space $E, Y$ a subspace of $X$, and $q$ a linear mapping from $Y$ into $E$ such that $q \leq p$ on $Y$. Then there exists a linear mapping $g$ from $X$ into $Y$ such that $g=q$ on $Y$ and $g \leq p$ on $X$.

Proof. Let $\phi$ be a mapping from $X$ into $E$ defined by

$$
\phi(x)=\inf \{p(x+t y)-t q(y) t \geq 0, y \in K\}
$$

for any $x \in X$. By Lemma 9, $\phi$ is a sublinear mapping such that $\phi \leq p$ on $X$. By Corollary 8 , there exists a linear mapping $g$ such that $g \leq \phi$ on $X$. Then putting $K=Y$ in Lemma 9, we have $g \leq p$ on $X$ and $q \leq g$ on $Y$. Since $Y$ is a subspace, for any $y \in Y$, we have $-y \in Y$. Then $q(-y) \leq g(-y)$. Since $q$ and $g$ are linear, we have $-q(y) \leq-g(y)$. Then $g \leq q$ on $Y$. Thus $g=q$ on $Y$. 
Moreover, by Corollary 8, we obtain the Mazur-Orlicz theorem in a Dedekind complete partially ordered vector space. For the case that $E$ is a Dedekind complete Riesz space, see $[1,15]$.

Theorem 11. Let $p$ be a sublinear mapping from a vector space $X$ into a Dedekind complete partially ordered vector space $E$. Let $\left\{x_{j} \mid j \in J\right\}$ be a family of elements of $X$ and $\left\{y_{j} \mid j \in J\right\}$ a family of elements of $E$. Then the following (1) and (2) are equivalent.

(1) There exists a linear mapping $g$ from $X$ into $E$ such that $g \leq p$ on $X$ and $y_{j} \leq g\left(x_{j}\right)$ for any $j \in J$.

(2) For any natural number $n$, nonnegative real numbers $\alpha_{1}, \alpha_{2}, \ldots, \alpha_{n} \geq 0$ and $j_{1}, j_{2}, \ldots, j_{n} \in J$, one has

$$
\sum_{i=1}^{n} \alpha_{i} y_{j_{i}} \leq p\left(\sum_{i=1}^{n} \alpha_{i} x_{j_{i}}\right) \text {. }
$$

Proof. Let $\alpha_{1}, \alpha_{2}, \ldots, \alpha_{n} \geq 0$ and $j_{1}, j_{2}, \ldots, j_{n} \in J$ for a natural number $n$. By (1), we have

$$
\begin{aligned}
\sum_{i=1}^{n} \alpha_{i} y_{j_{i}} & \leq \sum_{i=1}^{n} \alpha_{i} g\left(x_{j_{i}}\right) \\
& =g\left(\sum_{i=1}^{n} \alpha_{i} x_{j_{i}}\right) \leq p\left(\sum_{i=1}^{n} \alpha_{i} x_{j_{i}}\right) .
\end{aligned}
$$

Thus (2) is established.

Next by (2), for any $x \in X$, we have

$$
\begin{aligned}
\sum_{i=1}^{n} \alpha_{i} y_{j_{i}} & \leq p\left(\sum_{i=1}^{n} \alpha_{i} x_{j_{i}}\right)=p\left(x+\sum_{i=1}^{n} \alpha_{i} x_{j_{i}}-x\right) \\
& \leq p\left(x+\sum_{i=1}^{n} \alpha_{i} x_{j_{i}}\right)+p(-x), \\
-p(-x) & \leq p\left(x+\sum_{i=1}^{n} \alpha_{i} x_{j_{i}}\right)-\sum_{i=1}^{n} \alpha_{i} y_{j_{i}}
\end{aligned}
$$

for any natural number $n, \alpha_{1}, \alpha_{2}, \ldots, \alpha_{n} \geq 0$ and $j_{1}, j_{2}, \ldots$, $j_{n} \in J$. Put

$$
\begin{array}{r}
p_{0}(x) \\
=\inf \left\{p\left(x+\sum_{i=1}^{n} \alpha_{i} x_{j_{i}}\right)-\sum_{i=1}^{n} \alpha_{i} y_{j_{i}} \mid n \in N,\right. \\
\left.\alpha_{i} \geq 0, j_{i} \in J, i=1,2, \ldots, n\right\}
\end{array}
$$

for $x \in X$, where $N$ is the set of all natural numbers. By Lemma 3, $p_{0}$ is well defined. Since $p$ is sublinear, $p_{0}$ is also sublinear. Thus by Corollary 8 , there exists a linear mapping $g$ from $X$ into $E$ such that $g \leq p_{0}$ on $X$. Since $p_{0}\left(-x_{j}\right) \leq$ $p\left(-x_{j}+x_{j}\right)-y_{j}=-y_{j}$, we have

$$
y_{j} \leq-p_{0}\left(-x_{j}\right) \leq-g\left(-x_{j}\right)=g\left(x_{j}\right) \text {. }
$$

Since $p_{0}(x) \leq p(x)$ for any $x \in X$, we have $g(x) \leq p(x)$ for any $x \in X$. Thus (1) is established.

\section{Acknowledgment}

The authors would like to express their hearty thanks to Professor Koji Aoyama for careful reading and many valuable suggestions.

\section{References}

[1] R. Cristescu, Topological Vector Spaces, Editura Academiei, Bucharest, Romania, 1977, Translated from the Romanian by Mihaela Suliciu.

[2] P. M. Nieberg, Banach Lattices, Springer, Heidelberg, Germany, 1991.

[3] A. C. Zannen, Riesz Spaces II, North Holland, Amsterdam, The Netherlands, 1984.

[4] N. Hirano, H. Komiya, and W. Takahashi, "A generalization of the Hahn-Banach theorem," Journal of Mathematical Analysis and Applications, vol. 88, no. 2, pp. 333-340, 1982.

[5] S. Kakutani, "Two fixed-point theorems concerning bicompact convex sets," Proceedings of the Imperial Academy, vol. 14, no. 7, pp. $242-245,1938$.

[6] C. D. Aliprantis and O. Burkinshaw, Locally Solid Riesz Spaces with Applications to Economics, vol. 105 of Mathematical Surveys and Monographs, American Mathematical Society, Providence, RI, USA, 2nd edition, 2003.

[7] B. A. Davey and H. A. Priestley, Introduction to Lattices and Order, Cambridge University Press, New York, NY, USA, 2nd edition, 2002.

[8] M. M. Fel'dman, "Sufficient conditions for the existence of supporting operators for sublinear operators," Sibirskii Matematičeskič Žurnal, vol. 16, pp. 132-138, 1975 (Russian).

[9] T. Kawasaki, M. Toyoda, and T. Watanabe, “The Hahn-Banach theorem and the separation theorem in a partially ordered vector space," Journal of Nonlinear Analysis and Optimization, vol. 2, no. 1, pp. 111-117, 2011.

[10] W. A. J. Luxemburg and A. C. Zaanen, Riesz Spaces I, North Holland, Amsterdam, The Netherlands, 1971.

[11] N. Bourbaki, Topologie Générale, Hermann, Paris, France, 1940.

[12] W. A. Kirk, Fixed Point Theory: A Brief Survey, Universidas de Los Andes, Mérida, Mexico, 1990.

[13] H. Kneser, "Eine direkte ableitung des zornschen lemmas aus dem auswahlaxiom," Mathematische Zeitschrift, vol. 53, pp. 110$113,1950$.

[14] T. C. Lim, "On minimal (maximal) common fixed points of a commuting family of decreasing (increasing) maps," Differential and Difference Equations and Applications, pp. 683-684, 2006.

[15] A. L. Peressini, Ordered Topological Vector Spaces, Harper \& Row, New York, NY, USA, 1967. 


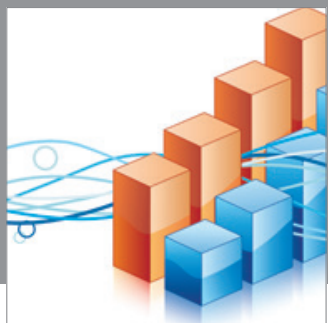

Advances in

Operations Research

mansans

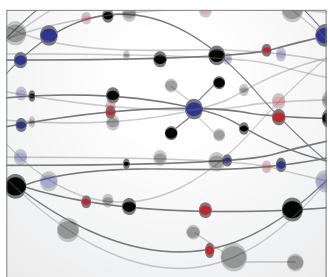

The Scientific World Journal
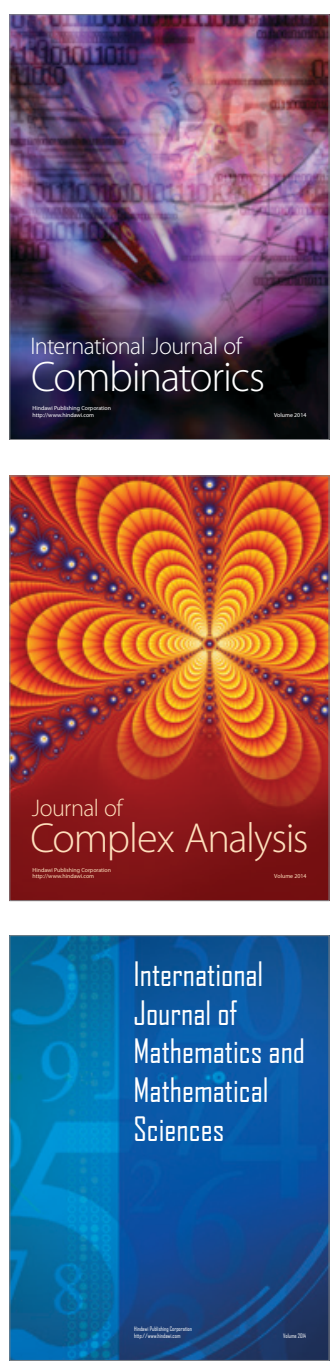
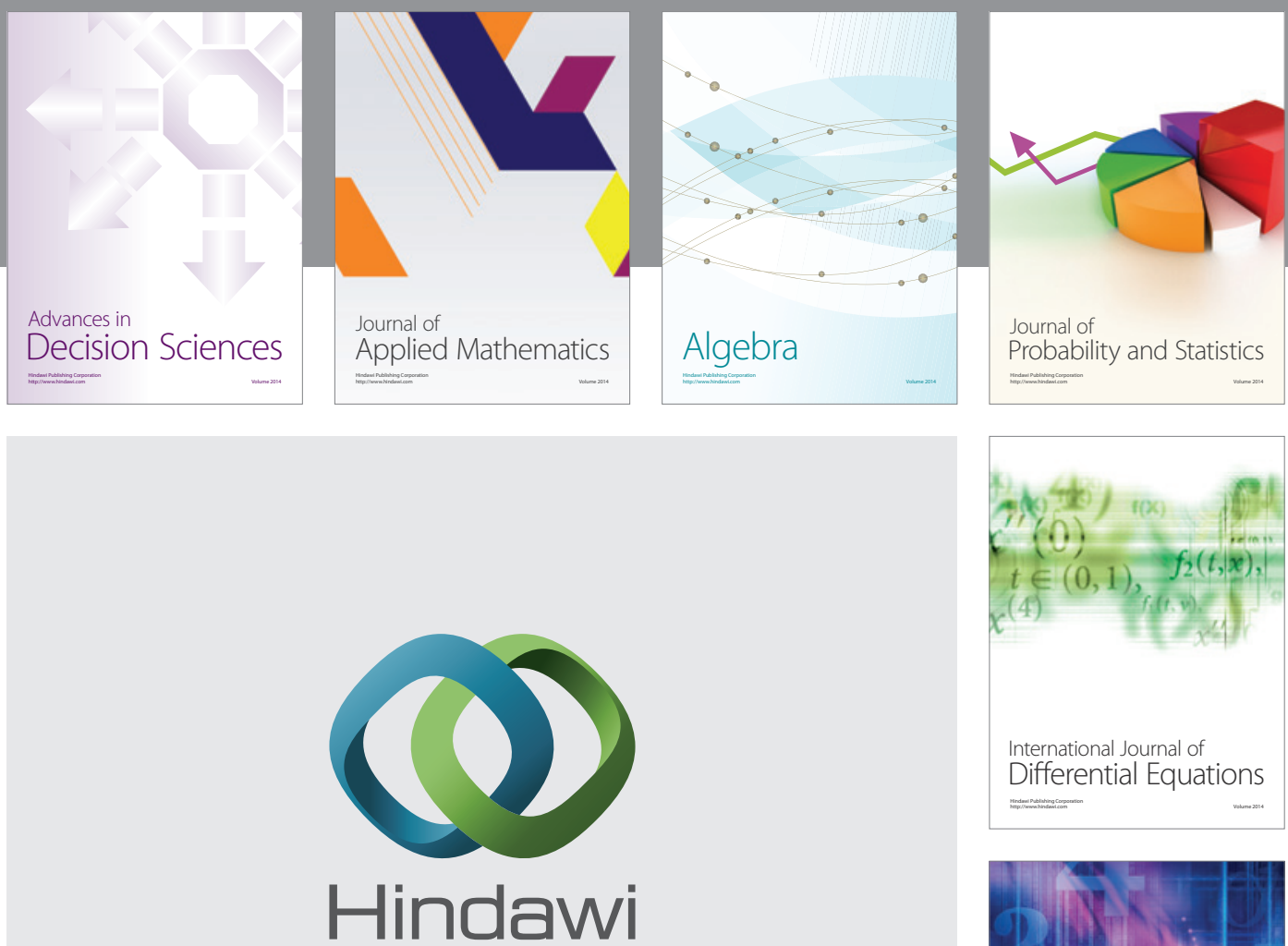

Submit your manuscripts at http://www.hindawi.com
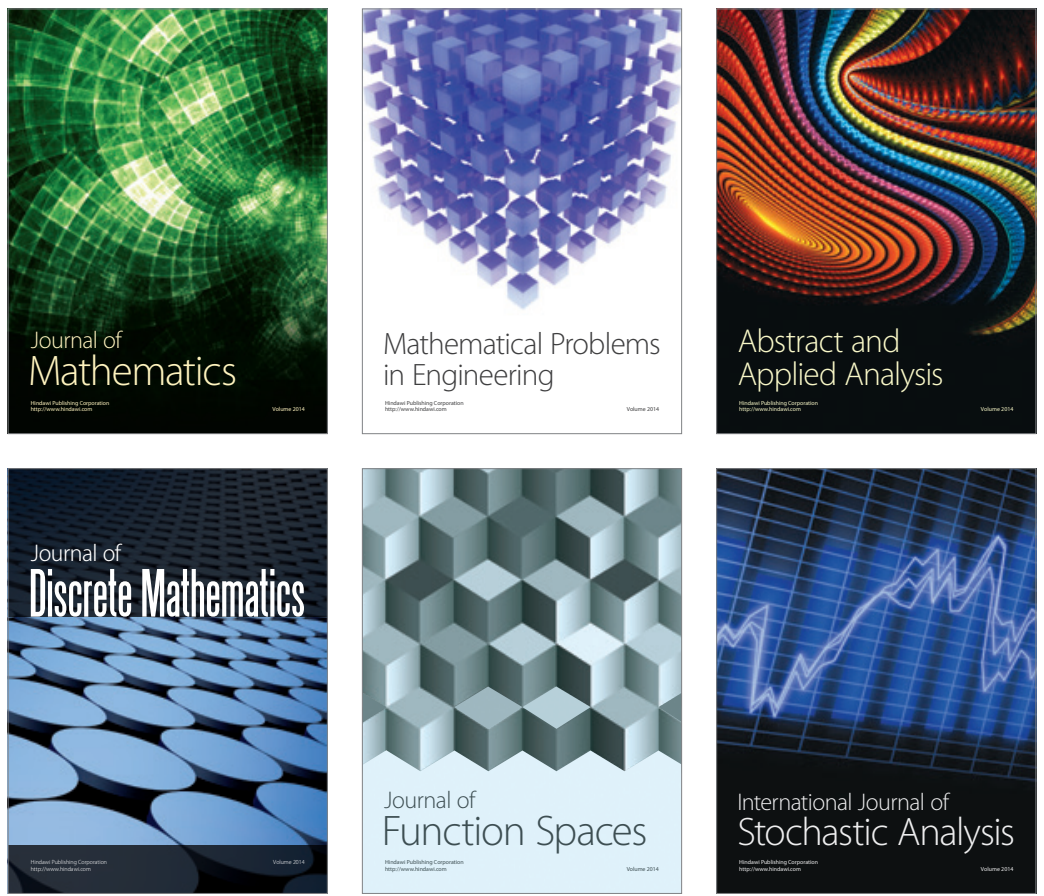

Journal of

Function Spaces

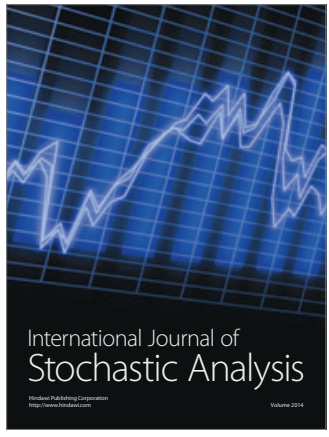

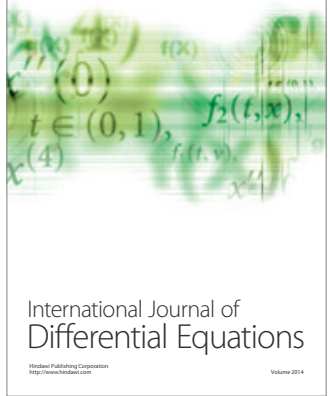
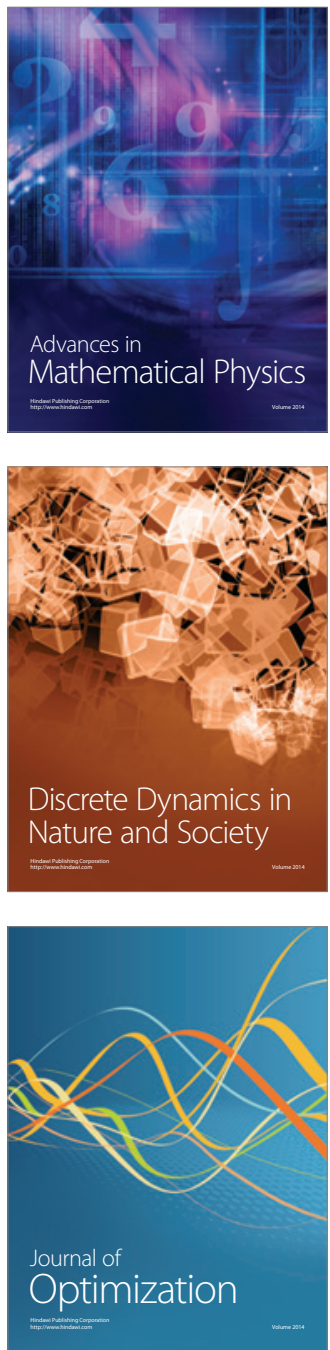